\title{
Evaluación de la toxicidad del extracto metanólico de hojas de Passiflora edulis Sims (maracuyá), en ratas
}

\author{
Passiflora edulis Sims (passion fruit) leaves methanol extract toxicity in rats
}

\author{
Juan Rojas ${ }^{1}$, David Díaz ${ }^{2}$ \\ ${ }^{1}$ Instituto de Investigaciones Clínicas, Facultad de Medicina, Universidad Nacional Mayor de San Marcos. Lima, Perú. \\ ${ }^{2}$ Instituto de Patología, Facultad de Medicina, Universidad Nacional Mayor de San Marcos. Lima, Perú.
}

\begin{abstract}
Resumen
Objetivo: Determinar la toxicidad oral a dosis repetidas del extracto metanólico de las hojas de Passiflora edulis en ratas. Diseño: Estudio experimental. Institución: Instituto de Investigaciones Clínicas e Instituto de Patología, Facultad de Medicina, Universidad Nacional Mayor de San Marcos, Lima, Perú. Material biológico: Ratas albinas, adultas hembras y machos, y hojas de Passiflora edulis. Métodos: Las hojas Passiflora edulis recolectadas en Trujillo fueron desecadas a $38^{\circ} \mathrm{C}$, pulverizadas y macerado con metanol. Se filtró, concentró y liofilizó. Se conformó dos grupos experimentales de 10 ratas ( 5 de cada sexo). Un grupo fue control, al cual se le administró agua destilada, y el otro fue grupo tratado, al cual se le administró extracto metanólico de las hojas de Passiflora edulis, en dosis de $200 \mathrm{mg} / \mathrm{kg}$, una vez/día, vía oral, durante 28 días. Se hizo diariamente observaciones clínicas a los animales, se controló el peso cada semana. Al final del experimento se realizó las determinaciones hematológicas y de bioquímica clínica y necropsia. Se extrajo los órganos para la determinación de su peso y estudio histopatológico. Principales medidas de resultados: Peso corporal, e indicadores hematológicos, bioquímicos e histopatológicos. Resultados: No se observó mortalidad ni alteraciones clínicas o hematológicas. La alanina aminotransferasa se incrementó muy ligeramente hasta 55,4 \pm 4,9 $\mathrm{U} / \mathrm{L}$, en machos ( $\mathrm{VN}=35,1-53,5)$, y 53,8 $\pm 3,9 \mathrm{U} / \mathrm{L}$ (VN $=28,8-46,0)$, en hembras. En el estudio histopatológico se observó en un caso microvesículas focal de hepatocitos y dos casos de focos de necrosis tubular. Conclusiones: La administración oral a dosis repetidas durante 28 días del extracto metanólico de las hojas de Passiflora edulis en ratas no es tóxica.
\end{abstract}

Palabras clave: Passiflora; toxicidad; extractos vegetales; ratas.

\begin{abstract}
Objective: To determine oral toxicity of Passiflora edulis leaves methanol extract repeated doses in rats. Design: Experimental study. Setting: Institute of Clinical Research and Institute of Pathology, Faculty of Medicine, Universidad Nacional Mayor de San Marcos, Lima, Peru. Biological material: Adult female and male albino rats, and Passiflora edulis leaves. Methods: Passiflora edulis leaves harvested in Trujillo were dried at $38^{\circ} \mathrm{C}$, powdered and macerated with methanol, filtered, concentrated and lyophilized. Two 10 rats (5 per sex) experimental groups were formed. One group was control that received distilled water and the other was the treated group that received Passiflora edulis leaves methanol extract in doses of $200 \mathrm{mg} / \mathrm{kg}$ once/day orally for 28 days. Clinical observations were done daily and the animals were weighed each week. At the end of the experiment we performed hematological, clinical biochemistry determinations and histology. Organs were extracted for determination of weight and histopathological study. Main outcome measures: Body weight, hematologic indicators, biochemical and histological analysis. Results: There was no mor tality or clinical and hematological abnormalities. Alanine aminotransferase increased very slightly to $55,4 \pm 4,9 \mathrm{U} / \mathrm{L}$ in males $(\mathrm{VN}=35,1-53,5)$ and 53,8 $\pm 3,9 \mathrm{U} / \mathrm{L}(\mathrm{VN}=28,8-46,0)$ in females. Histopathology revealed one case of focal hepatocytes microvesicles and two cases of renal tubular necrosis foci. Conclusions: Repeated oral dose administration of Passiflora edulis leaves methanol extract in rats for 28 days is not toxic.
\end{abstract}

Key words: Passiflora; toxicity; plant extracts; rats.

\section{INTRODUCCIÓN}

El género Passiflora comprende cerca de 500 especies, siendo la más grande la familia Passifloraceae, que está distribuida en regiones cálidas y tropicales del Nuevo Mundo; son mucho más raras en Asia, Australia y África tropical. Varias especies son cultivadas en los trópicos por sus frutos comestibles; los más ampliamente cultivados son Passiflora edulis Sims ${ }^{(1)}$.

Esta especie es cultivada ampliamente en países tropicales y subtropicales y existen dos variedades, Passiflora edulis Sims var. flavicarpa -cuyos frutos son amarillos, crece desde el nivel del mar hasta 1000 msnm- y, Passiflora edulis Sims var. purpúrea, con frutos color púrpura y que se adapta a zonas altas por encima de $1200 \mathrm{msnm}^{(2)}$. Se caracteriza por ser una planta leñosa perenne de hábito trepador y de rápido desarrollo, que puede alcanzar hasta $10 \mathrm{~m}$ de largo; las hojas son simples, alternas, con estípulas y un zarcillo en la axila, con márgenes aserrados; las flores son solitarias y axilares, fragantes y vistosas; el fruto es una baya esférica, globosa o elipsoide, que mide hasta $10 \mathrm{~cm}$ de diámetro y pesa hasta $190 \mathrm{~g}$, de color amarillo o purpúreo, con una pulpa muy aromática ${ }^{(3)}$.

El uso de Passiflora como una medicina fue elogiado por primera vez por un investigador español en el Perú, en $1569^{(1)}$. La información etnofarmacológica revela que Passiflora edulis Sims ha sido utilizado en medicina tradicional en diversas partes del mundo. En Sudamérica, se bebe la infusión de hojas y flores como sedante; la infusión de las partes aéreas es utilizada en el tratamiento de tétanos, epilepsia, insomnio e hipertensión ${ }^{(4)}$; además, se la indica como relajante muscular ${ }^{(5)}$, diurético, para tratar dolores estomacales, tumores intestinales y fiebre ${ }^{(6)}$.

Las investigaciones farmacológicas realizadas con $P$. edulis han demostrado que posee diversas propiedades. El extracto del fruto inhibió las enzimas que tienen actividad de endopeptidasas dependientes de zinc, las metaloproteinasas de matriz extracelular MMP-2 y MMP-9 involucradas en la invasión tumoral, metástasis y angiogénesis ${ }^{(7)}$, así como también inhibió la transformación neoplásica de células murinas $3 \mathrm{~T} 3 \mathrm{BALB} / \mathrm{c}$ tratadas con benzopireno ${ }^{(8)}$; el extracto de las hojas disminuyó la inflamación aguda y aumentó la proliferación fibroblástica, la colagenización y la neoformación capilar 
en la cicatrización de la vejiga de ratas (9); también, mostró una significativa actividad antiinflamatoria sobre pleuresía inducida por carragenina en ratones ${ }^{(10,11)}$; además, aumentó significativamente el número de ratones protegidos contra convulsiones inducidas por estricnina, de manera semejante al clonazepam ${ }^{(12)}$; y tuvo actividad antiviral contra herpes virus simple tipo $1, \mathrm{HSV}-{ }^{(13)}$. Por otra parte, se ha aislado un péptido antifúngico en las semillas de $P$. edulis ${ }^{(14)}$.

Respecto a la actividad de los extractos de $P$. edulis sobre el sistema nervioso central, existe información controversial. Algunos estudios demostraron efecto sedante ${ }^{(12)}$, ansiolítico ${ }^{(15,16)}$, tipo ansiolítico sin alterar la actividad motora ${ }^{(17)}$, tipo ansiolítico sin alterar el proceso de memoria ${ }^{(18)}$ y calmante tipo tranquilizante mayor ${ }^{(19)}$; sin embargo, otros investigadores afirmaron que no posee efecto ansiolítico ${ }^{(20)}$ ni efecto hipnótico-sedante; más bien, mostró efecto depresor no específico del sistema nervioso central ${ }^{(21)}$.

La Organización Mundial de la Salud apoya el uso adecuado de medicamentos a base de plantas y promueve el uso de los recursos que se han demostrado ser seguros y eficaces. Algunas plantas medicinales han resistido la prueba científica, pero otros simplemente son utilizados por razones tradicionales para proteger, restaurar o mejorar la salud. La mayoría de las plantas medicinales todavía necesita ser estudiada científicamente; a pesar de la experiencia obtenida de su uso tradicional en los últimos años, no debe ser ignorada ${ }^{(22)}$.

En un estudio previo, demostramos que el extracto etanólico de las hojas y el jugo del fruto de Passiflora edulis carecen de toxicidad aguda oral ${ }^{(23)}$. Solo existe un estudio de toxicidad con dosis repetidas de extracto acuoso de las hojas de esta planta realizado por Maluf ${ }^{(21)}$, en el que no obtuvo resultados concluyentes. Dado que se ha demostrado que $P$. edulis posee efecto antihipertensivo en ratas (23) y en humanos ${ }^{(24)}$ en donde es necesario un tratamiento prolongado; nos propusimos realizar esta investigación, con el objetivo de determinar la toxicidad oral del extracto metanólico de hojas de Passiflora edulis, a dosis repetidas, durante 28 días, en ratas.

\section{MÉTODOS}

Se realizó un estudio de tipo experimental, en el que se usó ratas albinas Holtzmann, hembras y machos, de 3 meses de edad, pesando en promedio $250 \mathrm{~g}$, adquiridas del Instituto Nacional de Salud. Los animales fueron mantenidos en el bioterio de la Facultad de Medicina de la Universidad Nacional Mayor de San Marcos (UNMSM), con alimento y agua ad libitum, temperatura promedio de $22^{\circ} \mathrm{C}$ y un ciclo de luz/oscuridad de $12 / 12$ horas. Se tuvo en cuenta las normas y procedimientos éticos para el manejo de animales de laboratorio establecidos internacionalmente ${ }^{(25)}$.

Las hojas de Passiflora edulis Sims fueron recolectadas en el Distrito de Moche, Provincia de Trujillo, Departamento de la Libertad, Perú. Una parte de la planta con hojas y flor fue llevada al Museo de Historia Natural de la Universidad Nacional Mayor de San Marcos, para la identificación taxonómica.

Las hojas fueron desecadas a $38^{\circ} \mathrm{C}$, en un horno con aire circulante, en el laboratorio de farmacología de la Facultad de Medicina de la UNMSM. El extracto metanólico de las hojas fue preparado según la técnica del CYTED, $1995^{(26)}$. El material seco y pulverizado de las hojas fue macerado con metanol durante 72 horas, a temperatura ambiente. Se filtró a través de filtro rápido (poros de 4,7 - 4,6 micras) y el filtrado se concentró a sequedad en un evaporador rotatorio (Büchi Rotavapor $($ ). El residuo fue disuelto en agua y liofilizado a $-45^{\circ} \mathrm{C}$ y 100 atmósferas de presión, durante 24 horas. El liofilizado fue conservado en un frasco color ámbar a $4^{\circ} \mathrm{C}$, hasta su uso.

La evaluación de la toxicidad oral a dosis repetidas durante 28 días se realizó según lo estipulado en el ensayo 407 de las directrices de la OECD (Organization for Economic Cooperation and Development) ${ }^{(27)}$. Se utilizó 20 ratas Holtzmann (10 de cada sexo); los animales fueron sometidos a un período de aclimatación de 5 días. El día antes del inicio del ensayo, se conformó los dos grupos experimentales de 10 animales ( 5 de cada sexo). Un grupo fue control (I), al cual se le administró vehículo (agua destilada), y el otro fue grupo tratado (II), al cual se le administró extracto metanólico de las hojas de Passiflora edulis, en dosis de 200 $\mathrm{mg} / \mathrm{kg}$, una vez al día, vía oral, durante 28 días. Se utilizó esta dosis debido a que en un estudio previo (datos no publicados) se determinó que el mejor efecto antihipertensivo del extracto metanólico se produjo con $200 \mathrm{mg} / \mathrm{kg} /$ día.

Se realizó diariamente observaciones clínicas a los animales, las mismas que incluyeron fundamentalmente cambios en la piel y el pelaje, ojos, membranas mucosas, ocurrencia de secreciones y excreciones, actividad autonómica (ej. lacrimación, piloerección, tamaño de pupila, patrón respiratorio inusual), cambios en el paso, postura, y respuesta a la manipulación, también como la presencia de movimientos tónicos o clónicos, estereotipias (ej. acicalamiento excesivo, dar vueltas repetitivas) o comportamiento extraño (ej. automutilación, caminar hacia atrás). En la cuarta semana de exposición, se evaluó la reactividad sensoria a los diferentes tipos de estímulos (ej. estímulo auditivo, visual y propioceptivo), la fuerza de agarre y la actividad motora.

Los animales fueron pesados al inicio del estudio y los días 7, 14, 21, así como al terminar el mismo. Al finalizar el estudio, se promedió el peso de los animales por semana y grupo, teniendo en cuenta el sexo, para observar cómo se comportó este parámetro durante el ensayo.

Se realizó determinaciones hematológicas y de bioquímica sanguínea, a los 28 días de exposición del extracto. La obtención de sangre se realizó por punción cardiaca, previo ayuno de $12 \mathrm{~h}$ de los animales. Se determinó el hemograma, alanina aminotransferasa (ALT), glucosa, colesterol total y triglicéridos.

Al finalizar la exposición, todos los animales fueron eutanizados con sobredosis de pentobarbital sódico (100 mg/ $\mathrm{kg}$ ) y sometidos a necropsia macroscópica detallada, la cual incluyó examen cuidadoso de la superficie externa del cuerpo, todos los orificios y las cavidades craneal, torácica y abdominal y sus contenidos. Se extrajo corazón, pulmones, riñones, bazo, hígado, ovario o testículos, separados de tejido adherente, y pesados. Estos órganos fueron fijados en formol al 10\%, embebidos en parafina, coloreados con 
hematoxilina eosina y se realizó el estudio microscópico correspondiente.

Los datos de las mediciones de peso corporal y órganos, así como de los indicadores hematológicos y bioquímicos fueron expresados como media aritmética \pm error estándar y sometidos a análisis de varianza (ANOVA) de un factor; y las diferencias entre los grupos fueron determinadas por la prueba post hoc de Scheffe; el nivel de significancia fijado fue $p<0,05$. Se utilizó el software estadístico SPSS 15,0 de Microsoft.

\section{RESULTADOS}

Durante el periodo de exposición de 28 días, no se observó síntomas tóxicos a la dosis administrada, tanto a nivel físico general como del comportamiento; tampoco se presentó mortalidad de los animales ni disminución del peso corporal, y la tendencia al aumento fue una constante durante el estudio en ambos sexos. Los datos fueron analizados de manera independiente para cada sexo y el análisis estadístico no mostró diferencias significativas del grupo tratado respecto al control en ambos sexos. Las figuras 1 y 2 muestran gráficamente el comportamiento del peso corporal en hembras y machos.

Los valores medios de los indicadores hematológicos se muestra en la tabla 1. No se observó alteraciones en la fórmula leucocitaria, observándose en todo momento células maduras acorde con su desarrollo, correspondiente con un comportamiento normal.

La tabla 2 muestra el comportamiento de los indicadores medios de química sanguínea. Para ambos sexos, los indicadores evaluados se encuentran dentro de los límites normales establecidos para la especie, excepto alanina aminotransferasa (ALT), que se incrementó muy ligeramente por encima de los valores normales (VN), alcanzando el valor de 55,4 $\pm 4,9$ $\mathrm{U} / \mathrm{L}$ en machos $(\mathrm{VN}=35,1-53,5)$ y 53,8 $\pm 3,9 \mathrm{U} / \mathrm{L}(\mathrm{VN}=28,8-46,0)$ en hembras, pero sin significación estadística.

No se produjo la muerte de animal alguno durante el estudio; por ello, todos fueron sacrificados al finalizar la

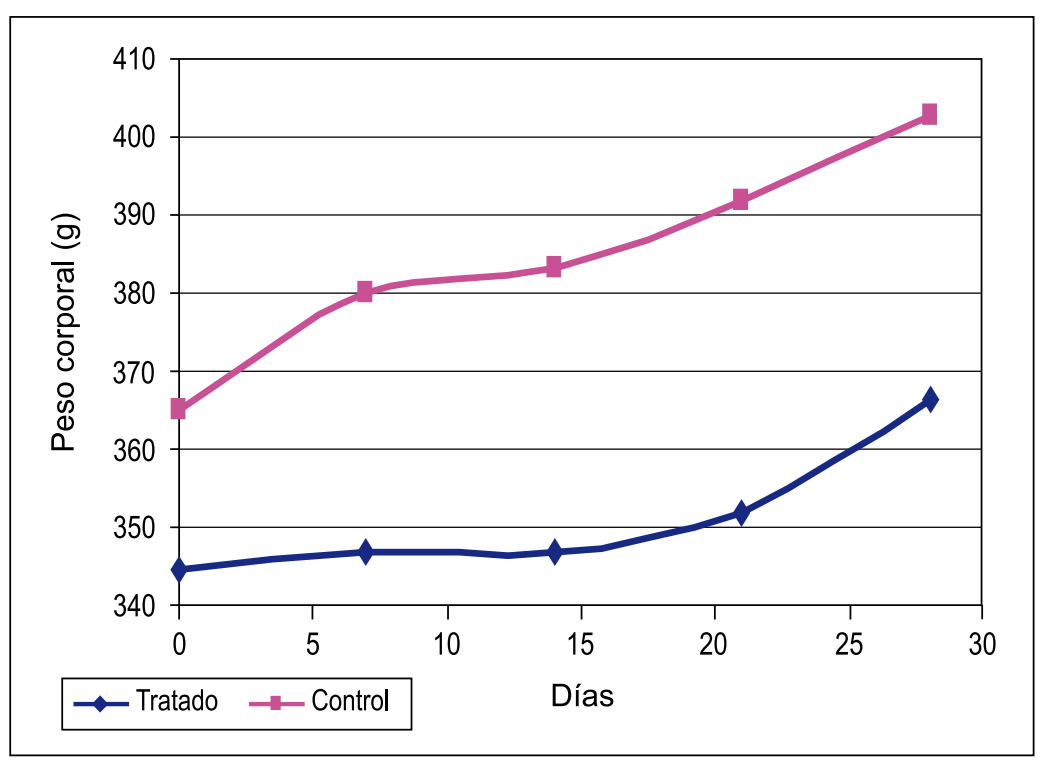

Figura 1. Comportamiento del peso corporal durante el estudio en machos.

investigación. Al realizar la necropsia, no se encontró lesiones macroscópicas atribuibles a la sustancia de ensayo.

Los valores de los pesos relativos de los órganos, en relación con el peso corporal (\%), no mostraron diferencias significativas respecto al control, tanto para machos como para hembras, tal como se muestra en la tabla 3.

En el estudio histopatológico de los órganos se observó un caso de microvesículas focal de hepatocitos; y, en otro caso, un infiltrado inflamatorio crónico mononuclear periportal focal y congestión vascular, como se muestra en la figura 3. En el riñón, se observó en dos casos focos de necrosis tubular, mostrado en la figura 4.

\section{DISCUSIÓN}

En la presente investigación, la administración por vía oral de dosis repetidas de extracto metanólico de las hojas de Passiflora edulis Sims, durante 28 días, no se asoció a mortalidad de las ratas ni signos tóxicos evidentes, además de no existir

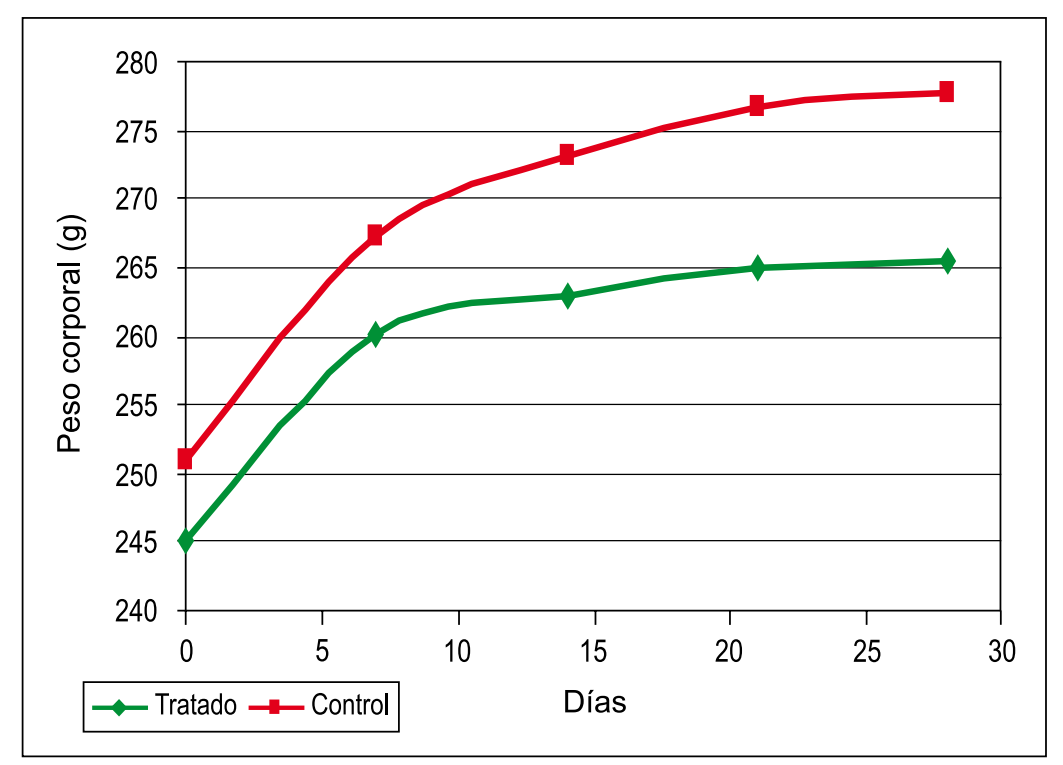

Figura 2. Comportamiento del peso corporal durante el estudio en hembras. 
Tabla 1. Variaciones en los parámetros hematológicos después de 28 días de tratamiento diario con $200 \mathrm{mg} / \mathrm{kg}$ de extracto metanólico de $P$. edulis.

\begin{tabular}{lcccccc}
\hline Grupo & $\begin{array}{c}\text { Leucocitos } \\
\left(\mathrm{x} 10^{3} / \mathrm{uL}\right)\end{array}$ & $\begin{array}{c}\text { Neutrófilos } \\
(\%)\end{array}$ & $\begin{array}{c}\text { Linfocitos } \\
(\%)\end{array}$ & $\begin{array}{c}\text { Monocitos } \\
(\%)\end{array}$ & $\begin{array}{c}\text { Eosinófilos } \\
(\%)\end{array}$ & $\begin{array}{c}\text { Basófilos } \\
(\%)\end{array}$ \\
\hline Machos & & & & & & \\
V.N. & $3,4-5,6$ & $22,8-28,3$ & $70,5-75,8$ & $0-4$ & $0-4$ & $0-1$ \\
Control & $4,2 \pm 0,5$ & $25,6 \pm 0,9$ & $72,8 \pm 1,1$ & $1,2 \pm 0,2$ & $0,8 \pm 0,4$ & $0,4 \pm 0,2$ \\
Tratado & $4,1 \pm 0,5$ & $26,0 \pm 0,9$ & $73,0 \pm 0,9$ & $1,4 \pm 0,5$ & $1,2 \pm 0,6$ & $0,2 \pm 0,1$ \\
Hembras & & & & & & \\
V.N. & $2,8-5,4$ & $21,1-27,3$ & $70,8-77,8$ & $0-4$ & $0-4$ & $0-1$ \\
Control & $4,4 \pm 0,5$ & $24,6 \pm 0,9$ & $74,0 \pm 1,2$ & $1,4 \pm 0,5$ & $0,8 \pm 0,4$ & $0,2 \pm 0,2$ \\
Tratado & $4,2 \pm 0,5$ & $23,2 \pm 1,2$ & $74,6 \pm 0,7$ & $1,8 \pm 0,6$ & $1,0 \pm 0,5$ & $0,6 \pm 0,2$ \\
\hline
\end{tabular}

Valores expresados como media aritmética \pm error estándar.

V.N. = valores normales.

Tabla 2. Variaciones en los parámetros de química sanguínea después de 28 días de tratamiento diario con $200 \mathrm{mg} / \mathrm{kg}$ de extracto metanólico de $P$. edulis.

\begin{tabular}{lcccc}
\hline Grupo & $\begin{array}{c}\text { Glucosa } \\
(\mathrm{mg} / \mathrm{dL})\end{array}$ & $\begin{array}{c}\text { Colesterol } \\
(\mathrm{mg} / \mathrm{dL})\end{array}$ & $\begin{array}{c}\text { Triglicéridos } \\
(\mathrm{mg} / \mathrm{dL})\end{array}$ & $\begin{array}{c}\text { Alanina amino- } \\
\text { transferasa }(\mathrm{U} / \mathrm{L})\end{array}$ \\
\hline Machos & & & & \\
V.N. ${ }^{*}$ & $95,7-147,7$ & $36,6-57,2$ & $33,3-65,5$ & $35,1-53,5$ \\
Control & $120,4 \pm 10,3$ & $50,6 \pm 3,1$ & $56,6 \pm 6,4$ & $46,8 \pm 4,1$ \\
Tratado & $118,4 \pm 10,1$ & $49,0 \pm 2,7$ & $55,2 \pm 6,3$ & $55,4 \pm 4,9$ \\
Hembras & & & & \\
V.N. & $112,9-170,9$ & $44,4-68,2$ & $38,1-66,1$ & $28,8-46,0$ \\
Control & $128,8 \pm 7,6$ & $58,8 \pm 5,2$ & $58,2 \pm 4,4$ & $40,6 \pm 3,3$ \\
Tratado & $126,6 \pm 6,2$ & $57,4 \pm 5,0$ & $57,8 \pm 5,9$ & $53,8 \pm 3,9$ \\
\hline
\end{tabular}

Valores expresados como media aritmética \pm error estándar.

V.N. = valores normales

Tabla 3. Variaciones del peso relativo de órganos después de 28 días de tratamiento diario con $200 \mathrm{mg} / \mathrm{kg}$ de extracto metanólico de $P$. edulis.

\begin{tabular}{lccccccc}
\hline Grupos & Corazón & Hígado & Bazo & Riñones & Pulmón & Ovarios & Testículos \\
\hline Machos & & & & & & & \\
Control & $0,47 \pm 0,02$ & $3,58 \pm 0,07$ & $0,36 \pm 0,04$ & $0,78 \pm 0,01$ & $0,91 \pm 0,05$ & - & $0,41 \pm 0,06$ \\
Tratado & $0,44 \pm 0,02$ & $3,67 \pm 0,10$ & $0,37 \pm 0,04$ & $0,77 \pm 0,03$ & $0,91 \pm 0,07$ & - & $0,43 \pm 0,04$ \\
Hembras & & & & & & & \\
Control & $0,38 \pm 0,01$ & $3,02 \pm 0,04$ & $0,24 \pm 0,01$ & $0,63 \pm 0,01$ & $0,85 \pm 0,09$ & $1,03 \pm 0,05$ & - \\
Tratado & $0,39 \pm 0,01$ & $3,16 \pm 0,06$ & $0,28 \pm 0,02$ & $0,65 \pm 0,02$ & $0,99 \pm 0,13$ & $1,10 \pm 0,04$ & - \\
\hline
\end{tabular}

Valores expresados como media aritmética \pm error estándar.

Diferencias no significativas respecto al control, tanto para machos como para hembras, analizados por ANOVA de un factor y una prueba post hoc de Scheffe.

Peso relativo $=100 \times$ peso del órgano/peso corporal.

alteración alguna de parámetros físicos y de comportamiento evaluados.

El peso corporal es un indicador de gran importancia, pues está involucrado en una serie de cambios orgánicos en diferentes etapas de la vida. Es por ello que una variación en su valor sugiere algún efecto adverso de drogas o químicos y se considera significativa si hay una disminución de más de $10 \%$ del peso corporal inicial ${ }^{(28)}$. Este indicador, en este estudio, tuvo un comportamiento normal de ganancia de peso y no se encontró diferencias significativas con respecto al control (figuras 1 y 2 ).
Los exámenes hematológicos y de química sanguínea son de gran valor en los ensayos toxicológicos a largo plazo, ya que los mismos son indicativos del alcance y profundidad de un daño. Además, los resultados alcanzados pueden ser correlacionados con los posibles daños sobre un órgano específico ${ }^{(29)}$. Los valores obtenidos en los indicadores hematológicos no mostraron alteraciones importantes; las variaciones encontradas se encuentran dentro del rango establecido por el grupo control y por la literatura especializada ${ }^{(30)}$. Sin embargo, dentro de los indicadores bioquímicos se observó un incremento muy leve en los valores de alanina aminotransferasa (ALT), por encima del valor normal (tabla 2), en las ratas sujetas a experimentación. La enzima ALT es citosólica y se encuentra en concentraciones más altas en hepatocitos, de manera que el incremento en el suero podría ser debido a modificaciones de la permeabilidad transmembrana o daño celular; por eso, es considerado como un indicador altamente sensible de hepatotoxicidad ${ }^{(31)}$. En este caso particular, dicho incremento no fue estadísticamente significativo, lo cual nos indicaría falta de toxicidad a este nivel.

El estudio microscópico del hígado reveló en un caso la presencia de microvesículas focal de hepatocitos y en otro, infiltrado inflamatorio crónico mononuclear periportal focal y congestión vascular (figura 3), lo cual guarda relación y podría ser la causa del leve incremento de los niveles séricos de ALT (tabla 2). Además, en el riñón se observó focos de necrosis tubular en dos casos del total de diez animales tratados (figura 4). Existe la posibilidad que las saponinas triterpenoidales presentes en las hojas de Passiflora edulis, demostrado por Yoshikawa y col. ${ }^{(32)}$ y confirmado por nosotros en un estudio fitoquímico preliminar ${ }^{(33)}$, puedan ser responsables del efecto sobre el hígado, ya que en una investigación toxicológica, en la que la composición de la muestra fue principalmente saponinas triterpenoidales, se sugirió que estas serían la causa del efecto tóxico sobre el hígado, posiblemente porque la mayoría de saponinas tienen una estructura química similar a las hormonas esteroides ${ }^{(34)}$; es conocido el efecto hepatotóxico de los estrógenos ${ }^{(35)}$. 


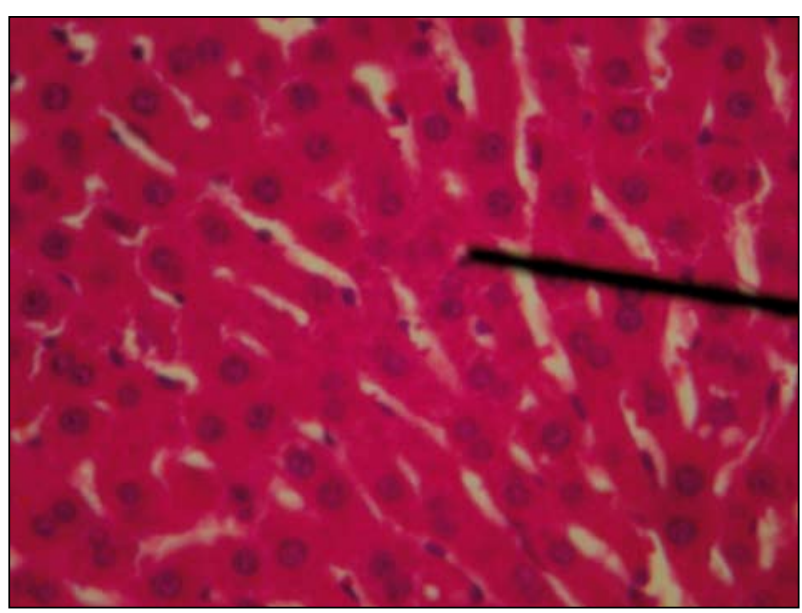

Figura 3A. Hígado normal (grupo control). Coloración HE 10x.

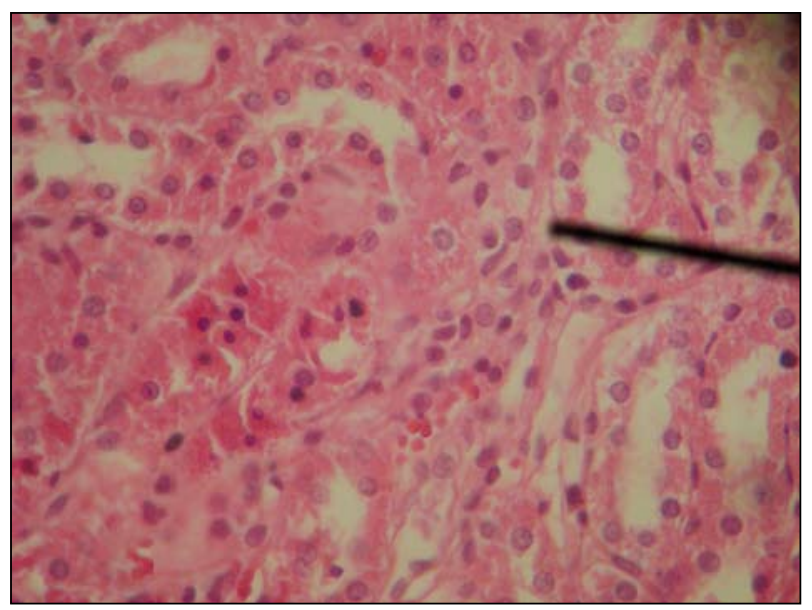

Figura 4A. Riñón normal (grupo control). Coloración HE 10x.

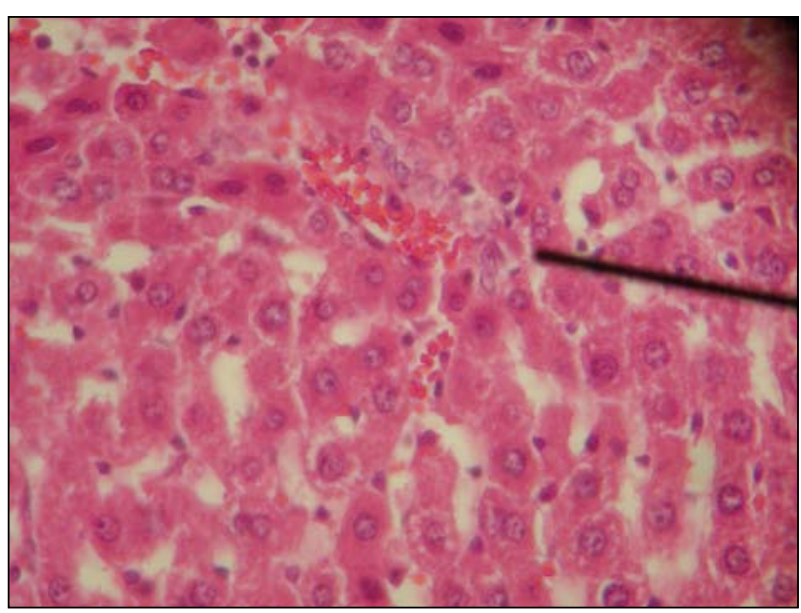

Figura 3B. Hígado con infiltrado inflamatorio crónico mononuclear periportal focal y congestión vascular.

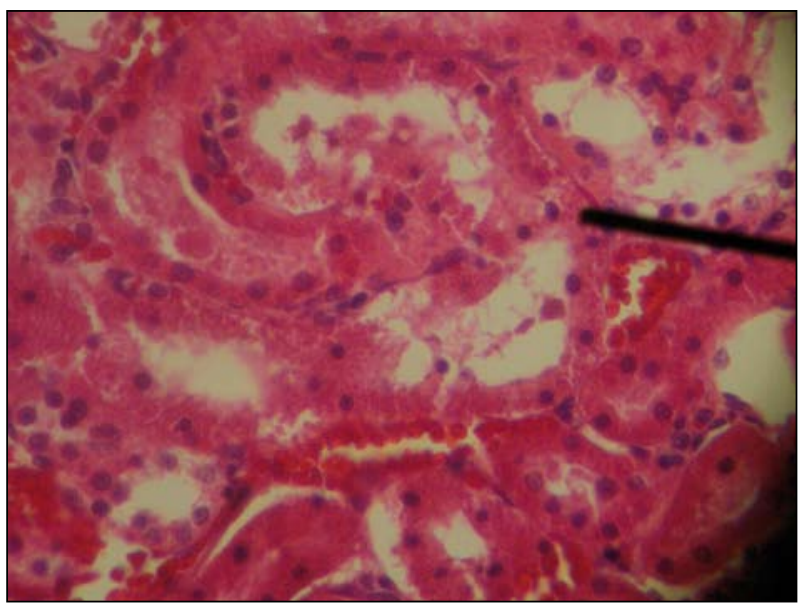

Figura 4B. Nefritis crónica focal con necrosis tubular focal (grupo tratado). Coloración HE 40x.
En Brasil, Maluf (21) evaluó en ratas dos muestras de hojas de Passiflora edulis en forma de extracto acuoso, administrándoles por vía oral, una procedente de Estado de Sao Paulo y la otra de Río Grande do Norte; la primera lo hizo a 60 días y la segunda hasta 90 días. En la primera, observó incremento de gama glutamil transpeptidasa (GGT) sérico (enzima que se eleva en enfermedades hepáticas colestásicas) y disminución del peso corporal, lo que no fue evidenciado con la muestra de Río Grande, la que fue evaluada aún a mayor tiempo. El autor busca explicar sus hallazgos argumentando que el aumento de GGT podría ser por un fenómeno de inducción enzimática y que los factores medio ambientales influyen en la variedad y cantidad de me- tabolitos secundarios que determinarían la variación de efectos encontrados en las muestras estudiadas de Passiflora edulis. Si bien la presente investigación guarda ligera relación con los resultados obtenidos por Maluf, tratándose de estudios de corto plazo, se debería ampliar los estudios de toxicidad crónica para aclarar el perfil toxicológico de esta planta, tal como lo sugieren los investigadores ${ }^{(21,36)}$.

No obstante que en la evaluación de la toxicidad aguda oral (dosis única) de Passiflora edulis, la dosis letal media (DL50) estimada, tanto para el extracto etanólico de las hojas como para el jugo de Passiflora edulis, supera ampliamente los $2000 \mathrm{mg} / \mathrm{kg}^{(23)}$, que según las normas de la Comunidad Europea ${ }^{(37)}$ para la clasificación de la toxicidad aguda oral es considerada no clasificado (no tóxico), la administración de dosis repetidas podría afectar hígado y riñón; sin embargo, en esta investigación, la administración oral diaria del extracto metanólico de las hojas de Passiflora edulis durante 28 días no mostró toxicidad evidente en ratas, lo cual guarda estrecha relación con el estudio clínico recientemente publicado, donde no se observó toxicidad en pacientes hipertensos que recibieron tratamiento diario con el jugo liofilizado del fruto de Passiflora edulis durante 28 días ${ }^{(24)}$.

En condiciones experimentales, la administración oral a dosis repetidas durante 28 días del extracto metanólico de las hojas de Passiflora edulis, en ratas, no mostró toxicidad fisiológica, morfológica, neurológica, de comportamiento, 
bioquímica sanguínea ni a nivel anatomopatológico.

\section{REFERENCIAS BIBLIOGRÁFICAS}

1. Dhawan K, Dhawan S, Sharma A. Passiflora: a review update. J Ethnopharmacol. 2004;94:1-23.

2. Ministerio de Agricultura y Ganadería. Aspectos técnicos sobre cuarenta y cinco cultivos agrícolas de Costa Rica. Costa Rica: Dirección General de Investigación y Extensión Agrícola; 1991. p. 30-2.

3. Galindo F, Villavicencio M. Maracuyá. Seminario de Agronegocios. Lima: Universidad del Pacífico; 2000.

4. Desmarchelier C, Witting F. Setenta plantas medicinales de la Amazonía peruana. $1^{\text {a }}$ ed. Lima: Gráfica Bellido; 2000. p. 187-90.

5. Brack A. Diccionario enciclopédico de plantas útiles del Perú. Cusco, Perú: Ed. Centro de Estudios Regionales Andinos Bartolomé de las Casas; 1999. p. 368-70.

6. Natural Products Alert (NAPRALERT SM). Profile for Passiflora edulis. Chicago: The board of trustees of the University of Illinois; 2001.

7. Puricelli L, Dell'Aica I, Sartor L, Garbisa S, Caniato R. Preliminary evaluation of inhibition of matrixmetalloprotease MMP-2 and MMP-9 by Passiflora edulis and $P$. foetida aqueous extracts. Fitoterapia. 2003;74(3):302-4.

8. Rowe C, Nantz M, Deniera C, Green K, Talcott S, Percival S. Inhibition of neoplastic transformation of benzo[alpha]pyrene-treated BALB/c 3T3 murine cells by a phytochemical extract of passion fruit juice. J Med Food. 2004;7(4):402-7.

9. Gonçalves A, Martins 0, Ligocki A, Tâmbara R, De Almeida C, Arnulf T, et al. Efeito do extrato de Passiflora edulis (maracujá) na cicatrização de bexiga em ratos:estudo morfológico. Acta Cirúrgica Brasileira. 2006;21(Supl. 2):3-8.

10.Vargas A, Geremias D, Provensi G, Fornari P, Reginatto F, Gosmann G, et al. Passiflora alata and Passiflora edulis spray-dried aqueous extracts inhibit inflammation in mouse model of pleurisy. Fitoterapia. 2007;78(2):112-9.

11. Montanher A, Zucolotto S, Schenkel E, Fröde T. Evidence of anti-inflammatory effects of Passiflora edulis in an inflammation model. J Ethnopharmacol. 2007;109(2):281-8.

12. Ngo E, Ngahb E, Ekoundic C, Dongc C, Ayissi R, Rakotonirinac $S$, et al. Sedative and anticonvulsant properties of passiflora edulis dried leaves decoction in mice. Afr J Trad CAM. 2004;1:63-71.

13. Müller V, Chávez J, Reginatto F, Zucolotto S, Niero $S$, Navarro D, et al. Evaluation of antiviral activity of south american plant extracts against herpes simplex virus type 1 and rabies virus. Phytother Res. 2007;21:970-4.

14. Pelegrini P, Noronha E, Muniz M, Vasconcelos I, Chiarello M, Oliveira J, et al. An antifungal peptide from passion fruit (Passiflora edulis) seeds with similarities to $2 \mathrm{~S}$ albumin proteins. Biochim Biophys Acta. 2006;1764(6):1141-6.

15.Petry R, Reginatto F, de-Paris F, Gosmann G, Salgueiro J, Quevedo J, et al. Comparative pharmacological study of hydroethanol extracts of Passiflora alata and Passiflora edulis leaves. Phytother Res. 2001;15:162-4.

16. Reginatto F, De-Paris F, Petra R, Quevedo J, González G, Gosmann G, et al. Evaluation of anxiolytic activity of spray dried powders of two south Brazilian Passiflora species. Phytother Res. 2006;20:348-51.

17. Coleta M, Batista M, Campos M, Carvalho R, Cotrim M, De Lima T, et al. Neuropharmacological evaluation of the putative anxiolytic effects of Passiflora edulis Sims, its sub-fractions and flavonoid constituents. Phytother Res. 2006;20:1067-73.

18. Barbosa P, Valvassori S, Bordignon C, Kappel V, Martins M, Gavioli E, et al. The aqueous extracts of Passiflora alata and Passiflora edulis reduce anxiety-related behaviors without affecting memory process in rats. J Med Food. 2008;11(2):282-8.

19. Bruschi M, Cardoso M, Milani H. Avaliaçao farmacológica de um extrato de Passiflora edulis variedade flavicarpa. Rev Ciênc Farm. 2002;23(2):263-76.

20. Dhawan K, Kumar S, Sharma A. Comparative biological activity study on Passiflora incarnata and P. edulis. Fitoterapia. 2001;72:698-702.

21. Maluf E, Barros M, Frochtengarten M, Benti R, Leite J. Assessment of the hypnotic/sedative effects and toxicity of Passiflora edulis aqueous extract in rodents and humans. Phytother Res.1991;5:262-6.

22. World Health Organization/Regional Office for the Western Pacific. Research Guidelines for Evaluating the Safety and Efficacy of Herbal Medicines. Manila: WHO; 1993.

23. Rojas J, Ronceros S, Palomino R, Tomás G, Chenguayen J. Efecto antihipertensivo y dosis letal 50 del jugo del fruto y del extracto etanólico de las hojas de Passiflora edulis (maracuyá) en ratas. An Fac med. 2006; 67(3):206-13.

24. Rojas J, Ronceros S, Palomino R, Salas M, Azañero $\mathrm{R}$, Cruz $\mathrm{H}$, et al. Efecto coadyuvante del extracto liofilizado de Passiflora edulis (maracuyá) en la reducción de la presión arterial en pacientes tratados con enalapril. An Fac med. 2009;70(2):103-8.

25. National Research Council. Guide for the care and use of laboratory animals. Washington, DC: National Academy Press; 1996.

26. CYTED. Programa Iberoamericano de Ciencia y Tecnología para el Desarrollo. Proyecto X-I. Búsqueda de principios bioactivos de plantas de la región. Manual de técnicas de investigación. Santa Fe de Bogotá. 1995. p. 220.

27. OECD. Guidelines for the Testing of Chemicals. Repeated dose 28-day oral toxicity study in rodents [Internet]. Paris: OECD; 1995 [citado 15 May 2008]. Disponible en: http://www.oecd.org

28. Ramesh T, Lee K, Lee H, Kim S. Acute oral toxicity study of Asiasari radix extract in mice. Int J Toxicol. 2007;26:247-51.

29. González Y, Scull I, Bada Ana, Fuentes D, González $B$, Arteaga M, et al. Ensayo de toxicidad a dosis repetidas durante 28 días del extracto acuoso de Cecropia peltata L. (yagruma) en ratas Cenp: SPRD. Rev Cubana Plant Med [Internet]. 2006 Jun [citado 2008 Jul 15]; 11 (2): Disponible en: http://scielo. sld.cu/scielo.php?script=sci_arttext\&pid=S102847962006000200005\&lng=es\&nrm=iso

30. Wolford S, Schroer R, Gohs F, Gallo P, Brodeck M, Falk $\mathrm{H}$, et al. Reference range data base for serum chemistry and hematology values in laboratory animals. J Toxicol Environm Health. 1986;18:16188.

31.Al-Habori M, Al-Aghbari A, Al-Mamary M, Baker M. Toxicological evaluation of Catha edulis leaves: a long term feeding experiment in animals. J Ethnopharmacol. 2002;83:209-17.

32. Yoshikawa K, Katsuta S, Mizumori J, Arihara S. Four cycloartane triterpenoids and six related saponins from Passiflora edulis. J Nat Prod. 2000;63(9):1229-34.

33. Rojas J. Estudio preclínico y clínico de la seguridad y actividad antihipertensiva de Passiflora edulis Sims (maracuyá). Tesis para optar el Grado de Doctor en Farmacia y Bioquímica. Universidad Nacional Mayor de San Marcos. Lima, Perú. 2009.

34.Burgos R, Hancke J, Wikman G. Toxicological assessment of Aralia mandshurica (Araliaceae) root extract after subchronic administration in rats. A biochemical and histological study. Phytother Res. 1994;8:1-9.

35. Yamamoto Y, Moore R, Hess H, Guo G, Gonzalez $\mathrm{F}$, Korach $\mathrm{K}$, et al. Estrogen receptor $\alpha$ mediates $17 \alpha$-ethynylestradiol causing hepatotoxicity. J Biol Chem. 2006;281(24):16625-31.

36.Zibadi S, Watson R. Passion fruit (Passiflora edulis): composition, efficacy and safety. EvidenceBased Integrative Medicine. 2004;1(3):183-7.

37. Unión Europea. Decisión 2000/368/CE de la Comisión: aproximación de las disposiciones legales, reglamentarias y administrativas en materia de clasificación, embalaje y etiquetado de las sustancias peligrosas. Diario Oficial. 2000; L136:108-25.

Manuscrito recibido el 12 de junio de 2009 y aceptado para publicación el 20 de agosto de 2009.

Correspondencia:

Dr. Juan Rojas Armas

Instituto de Investigaciones Clínicas - UNMSM

Hospital Nacional Dos de Mayo

Av. Grau cuadra 13. Parque Historia de la Medicina

Lima 1, Perú

Correo-e: jprojasarmas@yahoo.com 